Intensivmed 2009 · 46:462

DOI 10.1007/s00390-009-0103-7

Online publiziert: 2. Oktober 2009

(c) Springer-Verlag 2009

J. Graf ${ }^{1,2}$

${ }^{1}$ Medizinischer Dienst Deutsche Lufthansa AG LH-Basis FRA PM

Flughafen Bereich West, Aero Medical Center Frankfurt

${ }^{2}$ Medizinische Fakultät, Philipps-Universität Marburg

\title{
Innovationen in der Intensivmedizin
}

\section{Intensivmedizin ohne Grenzen}

Die Rubrik „Innovationen in der Intensivmedizin" wird im besten Sinne ein dynamisches Forum für alle Bereiche und alle Entwicklungen bzw. Neuerungen in der Intensiv- und Notfallmedizin bieten. In dieser Rubrik können (und sollen!) Grenzen überschritten und Traditionen infrage gestellt werden. Ein aktiver und hoffentlich auch kontroverser Gedankenaustausch zur Fortentwicklung unseres gemeinsamen Fachgebietes stellt das Ziel dieser Rubrik dar.

Das Spektrum möglicher Beiträge ist so groß und heterogen wie die Intensiv- und Notfallmedizin sich uns im Alltag präsentieren: neben technologischen Entwicklungen im Bereich des Monitorings, der Diagnostik oder der Therapie sind auch pharmakologische Innovationen, Neuerungen in der Versorgungsforschung oder Veränderungen im Qualitäts- und Risikomanagement von Interesse.

Der Stellenwert von Innovationen im Atemwegsmanagement, d. h. beispielsweise die Bedeutung der mobilen und handlichen Videolaryngoskopie im Gesamtkonzept des prä- und intraklinischen Managements des schwierigen Atemweges, wird uns in einer der nächsten Ausgaben der Zeitschrift „Intensivmedizin und Notfallmedizin“ beschäftigen. Spannend wären sicher auch Beiträge über konzeptionelle und pharmakologische Neuerungen im Bereich der Analgosedierung, veränderte Antikoagulationsregime bei der kontinuierlichen Nierenersatztherapie oder anderen extrakorporalen Verfahren oder die „Bodenhaltung“ Schädel-Hirntraumatisierter Intensivpatienten.

Die „Innovationen in der Intensivmedizin“ leben von Ihnen und mit Ihnen wir sind auf Ihre Impulse nicht nur angewiesen, sondern nehmen sie gerne auf. Sei es in Form von Fragen oder Themenvorschlägen oder aber auch - worüber wir uns natürlich außerordentlich freuen würden - wenn Sie als Autor eines Beitrags für diese Rubrik aktiv würden. Wir wünschen uns Originalarbeiten genauso wie Übersichtsartikel, Standpunkte oder spezifische Stellungnahmen.

Das Ziel der „Innovationen in der Intensivmedizin“" ist es, über den Tellerrand unseres Fachgebietes hinauszublicken und sinnvolle Aspekte aus anderen - auch primär nichtmedizinischen Bereichen - zu evaluieren und ggf. in die Versorgungskonzepte unserer Patienten mit einzubeziehen: eine Intensivmedizin ohne Grenzen!

PD Dr. J. Graf

\section{Korrespondenzadresse}

PD Dr. J. Graf

Medizinische Fakultät

Philipps-Universität Marburg

Baldingerstr., 35032 Marburg

jgraf@gmx.de 